\title{
Refuser, résister, guérir : plus que jamais d'actualité
}

S oixante ans de combats sans merci contre la maladie, avec, comme devise immuable, ces trois mots : refuser, résister, guérir. À l'origine de notre Association : la volonté et le désespoir de quelques parents, tous mus par un seul instinct, celui de sauver leurs enfants. C'est ainsi qu'en février 1958, neuf parents sont devenus les premiers administrateurs de l'Association Française contre la Myopathie; Yolaine de Kepper, acceptant d'en assurer la présidence.

Il faudra une décennie (1958-1968) pour exister et faire connaître. Les enfants étaient reçus en consultation par le Dr Jean Démos à l'hôpital Necker. La table d'examen était en fait un lit de plage déplié au milieu des appareils de laboratoire du Pr Robert Debré.

La $2^{e}$ décennie (1968-1978) verra en 1969 la reconnaissance de la myopathie comme affection de longue durée et la prise en charge à $100 \%$ des soins. Parallèlement, le réseau des militants se structure avec la mise en place de nos premières délégations régionales.

Une nouvelle dimension et une grande ambition caractérisent la $3^{e}$ décennie (1978-1988). Le 30 septembre 1986, lors du $2^{e}$ colloque sur les maladies neuromusculaires de Tours, le chercheur Anthony Monaco annonce la découverte du gène de la myopathie de Duchenne. Un an plus tard, le $1^{\text {er }}$ Téléthon - c'était le 4 décembre 1987, sur Antenne 2 - entamait une carrière sans précédent. Les moyens étaient là, enfin.

La 4écennie (1988-1998) a été à l'origine de l'épopée de la génétique et de la naissance, ou plutôt renaissance selon le Pr Michel Fardeau, de la myologie. Les premiers services régionaux de l'AFM voient le jour avec les premiers techniciens d'insertion. Au même moment ou presque, Généthon, le laboratoire financé par les dons des premiers Téléthons, est créé. Ce centre de génomique met la première carte physique à la disposition de la communauté scientifique internationale en 1992, suivie par la première carte génétique en 1996. En parallèle, pour que les malades et les familles puissent obtenir réponse à leurs questions,
l'AFM crée Allogènes en 1995 et soutient Orphanet en 1996. La bonne échelle pour nos malades étant le niveau européen, nous avons créé Eurordis en 1997 avec trois autres associations de malades. Pour comprendre les mécanismes de nos maladies neuromusculaires, il faudra construire avec des partenaires institutionnels, l'Institut de Myologie, un centre rassemblant dans un même hôpital, enseignement, recherche, soins et expertise médicale.

La $5^{e}$ décennie (1998-2008) sera marquée par la reconnaissance d'une stratégie et des premières victoires thérapeutiques. La Société Française de Myologie voit le jour en 2002. L'École d'Été de Myologie de Paris acquiert une réputation internationale et l'Institut de Myologie s'impose comme un acteur majeur dans la compétition mondiale en direction des thérapeutiques. En 2005, I-Stem est créé, contre vents et marées, grâce au soutien de l'Inserm et de l'AFM-Téléthon. Dix ans après, l'intérêt des cellules souches est prouvé pour la thérapie cellulaire.

Depuis, nous soutenons plus d'une trentaine d'essais cliniques en thérapie génique, thérapie cellulaire et en pharmacologie. Près de la moitié d'entre eux concernent des maladies neuromusculaires, les autres sont liées à d'autres maladies rares. Enfin en 2016, l'AFM-Téléthon et le fonds d'investissement SPI de Bpifrance créent YposKesi, le premier acteur industriel pharmaceutique français dédié aux maladies rares pour le développement et la production des produits de thérapies géniques et cellulaires.

Aujourd'hui, à l'heure du grand virage des traitements, à l'heure de la multiplication des essais, nous avons une obligation d'efficacité thérapeutique, à un prix juste et maîtrisé. Plus que jamais, nous devons nous mobiliser et agir ensemble pour cette nouvelle grande étape de notre histoire : GUÉRIR.

Refuse, resist, cure: now more than ever

Laurence Tiennot-Herment Présidente de l'AFM-Téléthon

LIENS D'INTÉRÊT

L'auteur déclare n'avoir aucun lien d'intérêt concernant les données publiées dans cet article. 